\title{
NREL Develops Test Facility and Test Protocols for Hydrogen Sensor Performance
}

Team: Safety Codes \& Standards Group, Hydrogen Technologies \& Systems Center

Accomplishment: The NREL Hydrogen Sensor Test Facility was recently commissioned for the quantitative assessment of hydrogen safety sensors (first reported in April 2010). Testing of sensors has started and is ongoing.

Test Apparatus: The Test Facility was designed to test hydrogen sensors under precisely controlled conditions. The apparatus can simultaneously test multiple sensors and can handle all common electronic interfaces, including voltage, current, resistance, controller area network, and serial communication. The lab is set up for around-theclock operation, and all tests can be run and monitored remotely via the Internet.

Test Plan: An NREL-developed test plan with well defined protocols has been implemented that subjects designated sensors to a rigorous regimen of procedures, in which sensor performance metrics are measured under prescribed gas composition and environmental stresses (temperature, pressure, and humidity extremes).

International Collaboration: In further support of hydrogen safety sensor development, NREL and the European Joint Research Centre's (JRC's) Institute for Energy in the Netherlands are collaborating via round-robin testing of representative commercial hydrogen detectors. This collaboration has been called the Sensor Interlaboratory

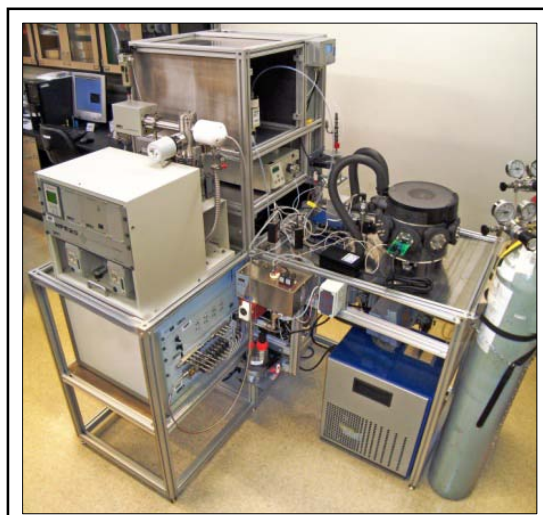

The NREL hydrogen safety sensor test facility (Robert Burgess/NREL) PIX 18240

Comparison (SINTERCOM) Project. Upon review and acceptance by JRC personnel, the NREL test plan was implemented for the SINTERCOM evaluations.

Manufacturer Support: The Sensor Laboratory provides manufacturers access to a state-of-the-art test facility for an independent, unbiased evaluation of their technologies. Currently, evaluations are performed under the auspices of SINTERCOM and at the request of manufacturers. Numerous manufacturers have contacted NREL to schedule access to the test facility. Data and an expert critique of performance metrics are shared with manufacturers. Through such interactions, NREL has provided manufacturers with recommendations to improve operation of their technologies to better meet end-users' needs.

Context: The U.S. Department of Energy (DOE) is developing hydrogen as a clean and renewable alternative to carbonbased fuels. One critical aspect for the safe and efficient deployment of hydrogen is the ability of chemical sensors to meet required performance specifications for the growing hydrogen infrastructure.

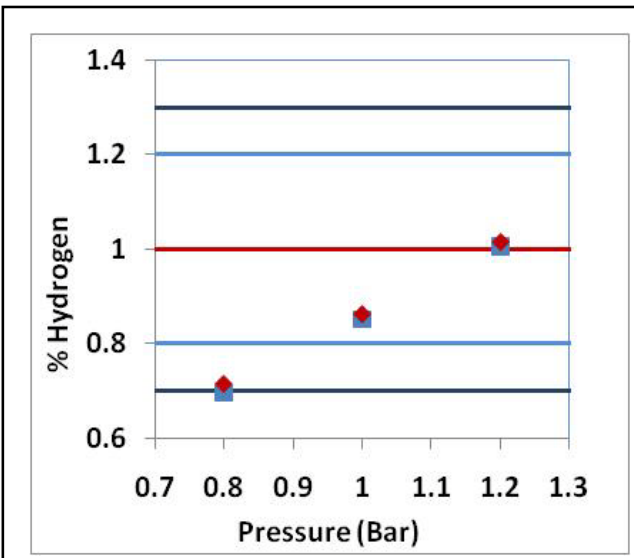

Pressure induced change on sensor output to a $1 \% \mathrm{H}_{2}$ test gas (-) as measured by JRC on Unit 1 ( $\bullet$ ) and NREL on Unit $2(\bullet)$ compared to the ISO (-) and vendor tolerances (-)
SINTERCOM Round-Robin Test: NREL and JRC are independently assessing various hydrogen detection technologies using mutually agreedupon test protocols based upon the NREL test plan. The first round of testing has been completed and results for pressure effects are shown. As mandated by SINTERCOM protocols, NREL and JRC have exchanged sensor units for the second round of evaluations, thus subjecting all units to evaluations at both laboratories. By independently testing the same sensors, both labs gain insight into their respective systems, facilitating improved testing capabilities, protocols, and data analysis. A review of SINTERCOM was held during an NREL site visit to JRC between March 29 and April 1, 2010. During the site visit, test procedures and results, methods for comparing test data, and proposed protocols for data analysis and presentation were discussed. Additional sensors for future assessment by SINTERCOM were also identified.

Applicable DOE Technical Targets: This work supports hydrogen safety sensor R\&D targets as specified in the DOE Multi-Year RD\&D Plan.

Significance of Accomplishment: The NREL sensor testing provides vendors access to a state-of-the art test facility for performance assessment and, through SINTERCOM, exposure to international markets. SINTERCOM provides an excellent technical basis for the development of a common codes and standards language, enabling consistent results from laboratory to laboratory. 